\title{
Review: long term psychotherapy in combination with antidepressants increases compliance and response rates in people with depression
}

Pampallona S, Bollini P, Tibaldi G, et al. Combined pharmacotherapy and psychological treatment for depression. A systematic review. Arch Gen Psychiatry 2004;61:714-19.

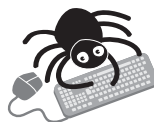

This article

contains extra text on the EBMH website

Does psychotherapy in combination with antidepressants increase compliance and response rates compared with antidepressants alone in people with depression?

\section{METHODS}

Design: Systematic review with meta-analysis.

Data sources: MEDLINE, Current Contents, PsycINFO, and the Cochrane Library (January 1990 to November 2002); hand searches of review articles, textbooks, treatment guidelines, and reference lists.

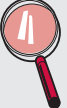

Study selection and analysis: Eligible studies were randomised clinical trials comparing antidepressants plus psychotherapy (problem solving, interpersonal, cognitive, behavioural, marital, and psychodynamic therapies) with antidepressant treatment alone. Exclusions: non-pharmacological intervention other than psychotherapy; subsequent publications from an already published trial (data subsets or long term follow up). Data were extracted on diagnosis, sex, age, type of antidepressant, type of intervention, and study duration. Study quality and duration were used to perform meta-regression analysis on random effect estimates.

Five

Outcomes: Response rate; dropout rate.

\section{MAIN RESULTS}

Sixteen studies met inclusion criteria. 910 people received psychotherapy plus antidepressants and 932 people received antidepressants alone (median female: 65\%; study length: 4-24 weeks; dose equivalent of imipramine hydrochloride: $100-269 \mathrm{mg} /$ day). Psychotherapy plus antidepressants significantly improved response rates compared with antidepressants alone in people with depression (see http://www.ebmentalhealth.com/supplemental for table). There were no significant differences in dropout rate between groups. Study length had no effect on response rate; however studies more than 12 weeks long had significantly reduced dropout rates.

\section{CONCLUSIONS}

Psychotherapy in combination with antidepressants significantly improves response rates in people with depression. For studies longer than 12 weeks, the combined therapy also significantly reduces dropout rates.

\section{NOTES}

Standardisation of antidepressant doses was carried out by conversion to the equivalent dose of imipramine hydrochloride. Rate difference analysis showed no change in non-response rate $(0.4 \%)$ and a significant reduction in dropout rate $(-12.9 \%)$ (see table).

For correspondence: Carmine Munizza, MD, Centro Studi e Ricerche Psichiatria, Piazza del Donatore di Sangue, 3, 10154 Torino, Italy; cmunizza@tin.it

Sources of funding: Centro Studi e Ricerche in Psichiatria, Instituto Superiore di Sanita, Rome and Ravizza Pharmaceuticals, Milan, Italy.

\section{Commentary}

A dherence to antidepressant medication (ADM) is becoming increasingly recognised as problematic. ${ }^{1} 2$ Nevertheless, randomised clinical trials of treatments for depression have focused almost exclusively on efficacy and have not systematically examined adherence. ${ }^{3}{ }^{4}$ According to Pampallona et al, the few controlled studies that have examined adherence involve interventions that are multilayered, thus obfuscating factors that affect adherence. Further, the authors report that these few controlled studies on ADM adherence do not systematically measure clinical outcomes such as remission. Thus, it is unclear which factors influence adherence and how it is related to clinical outcome.

The authors examined the effects on outcomes and adherence of combined treatment (pharmacotherapy plus psychotherapy) versus pharmacotherapy only by performing a meta-analysis on 16 randomised clinical trials. Each clinical trial included pharmacotherapy and pharmacotherapy plus psychotherapy conditions, as well as data on outcome measures.

The authors found that combined treatment reduced non-response to treatment, thus participants experienced some, if not total alleviation of depressive symptoms. Further, the addition of psychotherapy to pharmacotherapy increased participant retention in studies of 12 weeks or longer; however, there was no significant decrease in non-response rate above and beyond that observed during the first 12 weeks or in studies less than 12 weeks in length.

The authors point out that the precise causes of the improved outcomes achieved with combined treatment are unknown and future studies are needed. We agree with the authors that future research on this topic holds considerable promise. Our research on ADM treatment of depression is guided by the notion that the patient-pharmacotherapist relationship, a variable often studied in psychotherapy trials and considered crucial in achieving therapeutic outcomes, ${ }^{5}$ is largely overlooked in studies of adherence to ADM. It is our hope that research on psychological factors involved in ADM treatment adherence will help to further integrate the findings from the somewhat disparate depression treatment literature on psychosocial and biological approaches with the promise of providing further benefit to patients.

Christeine M Terry, BA and Robert J Kohlenberg, PhD University of Washington, Seattle WA, USA

1 National Institute of Mental Health (21 April 2003). Research on Adherence to Interventions for Mental Disorders. Program Announcement 03-111, 21 April 2003. Available at http://grants2.nih.gov/grants/guide/pa-files/ PA-03-111.html (accessed May 2004).

2 Katon W, Rutter C, Ludman EJ, et al. A randomized trial of relapse prevention of depression in primary care. Arch Gen Psychiatry 2001;58:241-7.

3 Keller MB, Hirschfeld RMA, Demyttenaere K, et al. Optimizing outcomes in depression: focus on antidepressant compliance. Int Clin Psychopharmacol 2002; 17:265-71

4 Gelenberg AJ, Trivedi MH, Rush AJ, et al. Randomized, placebo-controlled trial of nefazodone maintenance treatment in preventing recurrence in chronic depression. Biol Psychiatry 2003;54:806-17.

5 Carroll KM, Nich C, Rounsaville BJ. Contribution of the therapeutic alliance to outcome in active versus control psychotherapies. J Consult Clin Psychol 1997;65:510-14. 